\title{
Corneal Nerve Architecture in a Donor with Unilateral Epithelial Basement Membrane Dystrophy
}

\author{
Jiucheng He Haydee E.P. Bazan \\ Department of Ophthalmology and Neuroscience Center of Excellence, Louisiana State University Health Sciences \\ Center, School of Medicine, New Orleans, La., USA
}

\section{Key Words}

Epithelial basement membrane dystrophy - Corneal innervations - Immunofluorescence of corneal nerves • Recurrent erosion syndrome

\begin{abstract}
Background: Epithelial basement membrane dystrophy (EBMD) is by far the most common corneal dystrophy. In this study, we used a newly developed method of immunofluorescence staining and imaging to study the entire corneal nerve architecture of a donor with unilateral EBMD. Method: Two fresh eyes from a 56-year-old male donor were obtained; the right eye of the donor was diagnosed with EBMD and the left was normal. After slit lamp examination, the corneas were immunostained with anti- $\beta$-tubulin III antibody. Images were recorded by a fluorescent microscope equipped with a Photometrics digital camera using MetaVue imaging software. Results: The left cornea appeared normal as observed by slit lamp and stereomicroscope, but the right eye had numerous irregular geographic patches in the basement membrane. Immunofluorescence showed no difference in the stromal nerve distribution between the 2 eyes, but there were areas without innervations in the EBMD cornea. Subbasal nerve fibers also showed tortuous courses and fewer divisions. There was a significant decrease in the
\end{abstract}

density of subbasal nerve fibers and the number of terminals in the right eye. Conclusion: We show for the first time detailed nerve architecture in an EBMD cornea. Our results suggest that EBMD-induced abnormalities of basement membrane altered epithelial nerve architecture and decreased nerve density, contributing to the pathology of the disease.

Copyright $\odot 2013$ S. Karger AG, Basel

\section{Introduction}

Epithelial basement membrane dystrophy (EBMD), also known as Cogan's microcystic epithelial dystrophy or map-dot-fingerprint dystrophy, is the most common corneal dystrophy. It was first described by Vogt [1] in 1930, and later Cogan [2] (1964), Guerry [3] (1965) and Rodrigues [4] (1974) provided a thorough evaluation of the pathology. It has been estimated that EBMD affects nearly $42 \%$ of persons of all ages, and as many as $76 \%$ of persons worldwide who are over the age of 50 [5]. Patients with EBMD often experience painful chronic recurrent corneal erosion, photophobia and decreased vision, although some EBMD patients may be asymptomatic $[6,7]$.

Histologically, EBMD is characterized by the secretion of an anomalous basement membrane, part of which extends into the epithelial layer, and by intraepithelial mi-

\section{KARGER}

Fax +4161306 1234

E-Mail karger@karger.com

www.karger.com (c) 2013 S. Karger AG, Basel

$0030-3747 / 13 / 0494-0185 \$ 38.00 / 0$

Accessible online at:

www.karger.com/ore
Haydee E.P. Bazan

Department of Ophthalmology and Neuroscience Center of Excellence

Louisiana State University Health Sciences Center

2020 Gravier St., Suite D, New Orleans, LA 70112 (USA)

E-Mail hbazanl@lsuhsc.edu 
crocysts containing cellular debris associated with abnormalities of epithelial cell morphology [8-15].

In many cases, the poor adhesion of basal epithelium to abnormal lamellar material can produce the recurrent erosion observed in EBMD [16-19]. Corneal nerves are important for maintaining tear secretion and a healthy epithelium, and a decrease in nerve density could account for the development of corneal erosions [20,21].

Morphological abnormalities in epithelial cells and basement membrane associated with EBMD have been well studied by light, electron and, more recently, in vivo confocal microscopes [8-12, 19, 22-24], but little is known about corneal nerve architecture in the disease. Using in vivo confocal microscopy, changes in corneal nerves have been reported in different corneal dystrophies [23-28]. In patients with EBMD, pathological changes in the subbasal nerves with a decrease (and even absence in 1 patient) of the subbasal plexus have been reported [23], but these studies limit the examination to a small portion of the cornea. Here, we used a modified method of immunofluorescence and imaging $[29,30]$ to study the entire corneal nerve architecture in a donor with unilateral EBMD.

\section{Case Report}

This study was conducted according to the tenets of the Declaration of Helsinki. Two eyes from a 56-year-old male donor who died of heart attack were obtained from the National Disease Research Interchange. The eyes were kept in a wet chamber and shipped to our laboratory on ice. The donor had diagnosed EBMD in the right eye but had no other history of eye disease, contact lens wear, ocular surgery, or other systemic diseases that might have affected the cornea. Examination of the normal cornea with a portable digital slit lamp (Optotek Medical, Ljubljana, Slovenia) and a stereomicroscope (Nikon SMZ-1500) showed no abnormalities. In the EBMD eye, dark-field illumination (fig. 1a) showed numerous irregular geographically shaped, faint gray-white patches that appeared as fingerprint dots and ridge-like lesions. These structures were extensively present in the central area of the inferior quadrants of the cornea. Figure $1 \mathrm{~b}$ shows the frame area from figure 1a using oblique illumination. The irregular pathological foci are located in the basement membrane with sharp borders insinuating into the corneal epithelium. These fingerprint irregularities can be seen using the higher magnification shown in figure 1d; this was taken from the dashed frame area of figure la. Figure $1 \mathrm{c}$ is the dark-field image of figure $1 \mathrm{~d}$.

The time between death and fixation was $28 \mathrm{~h}$. Corneas were processed as previously described and stained with anti- $\beta$-tubulin III antibody (Tuj1, MMS-435P, 1:3000; Convance Antibody Services Inc., Berkeley, Calif., USA) in 1\% normal goat serum plus $0.15 \%$ Triton X-100 in $0.1 \mathrm{M} \mathrm{PBS}$ for $72 \mathrm{~h}$ at $4^{\circ} \mathrm{C}[29,30]$. Consecutive images were acquired by a fluorescence microscope (Nikon Eclipse TE200) equipped with a Photometrics digital camera (CoolSNAP ${ }^{\mathrm{TM}} \mathrm{HQ}$ ) using MetaVue imaging software. The imag- es, which were recorded on the same plane at adjoining points, were merged using Photoshop imaging software (Adobe, Mountain View, Calif., USA) and then pasted onto a Microsoft Office PowerPoint template to build the whole view of the corneal epithelial nerve architecture. To acquire the whole image of the stromal nerve structure, consecutive images were recorded by a stereoscopic zoom microscope (Nikon SMZ 1500) as previously reported $[29,30]$. Images were merged to build a complete view of corneal stromal nerves as described above.

No obvious difference was found in the number and distribution of stromal nerves between the 2 eyes (data not shown). Figure 2a shows the whole mount view of the epithelial nerve architecture of the normal cornea. Long epithelial nerve bundles run from the periphery to the center, converging into an area within the inferior nasal quadrant (vortex area). Highlighted images (fig. $2 \mathrm{~b}-\mathrm{d}$ ) show the details of the epithelial nerve architecture in the periphery, center and vortex areas. An intense array of nerve bundles coursed from the periphery towards the center. These long bundles divided into numerous smaller branches, which connected to each other to constitute the epithelial nerve network. Free nerve endings budding from the network innervated the epithelial cells (fig. 2e).

Figure $2 \mathrm{f}$ shows the whole mount view of the epithelial nerve distribution within the pathological area in the EBMD cornea. In comparison with the normal eye, the subbasal epithelial nerve density was obviously reduced, with many areas without innervations. There were significantly fewer divisions and connections between the nerve bundles, and the course of the nerves changed along the shapes of pathological loci. Highlighted images show the epithelial nerve distribution in pathological loci at the central (fig. $2 \mathrm{~g}$ ) and vortex (fig. $2 \mathrm{~h}$ ) areas; arrows show the pathological lesions. Topographical images in figure $2 \mathrm{i}$ show the detailed epithelial nerve architecture at different depths from superficial (I) to the subbasal layer of the epithelia (IV) in a finger-shaped lesion located in the central area. Epithelial nerve divisions and bundles run surrounding the lesion with some twisted nerves inside the lesion area. Fewer free nerve endings than found in the normal cornea are seen in I and II.

To compare the subbasal nerve densities within the central zone of the inferior quadrants of both eyes, a total of 36 images (about $42 \mathrm{~mm}^{2}$ ), acquired with a $10 \times$ lens from each eye, were calculated. A big difference in subbasal nerve density was found between the EMBD and the normal eye (fig.3a, b).

Nerve terminals in superficial epithelia were calculated by directly counting the number of free endings in each image and expressing them as the number of terminals $/ \mathrm{mm}^{2}$; a total of 20 images (about $6 \mathrm{~mm}^{2}$ ), acquired with a $20 \times$ lens from each eye, were calculated. There was an obvious decrease in nerve terminals in the EMBD eye (fig. 3c, d).

\section{Discussion}

Corneal dystrophies are disorders not associated with inflammation. Clinically they are classified according to the corneal layer involved [31]. Some dystrophies affect primary corneal epithelium and its basement membrane. EBMD, the most common dystrophy, is usually bilateral, 

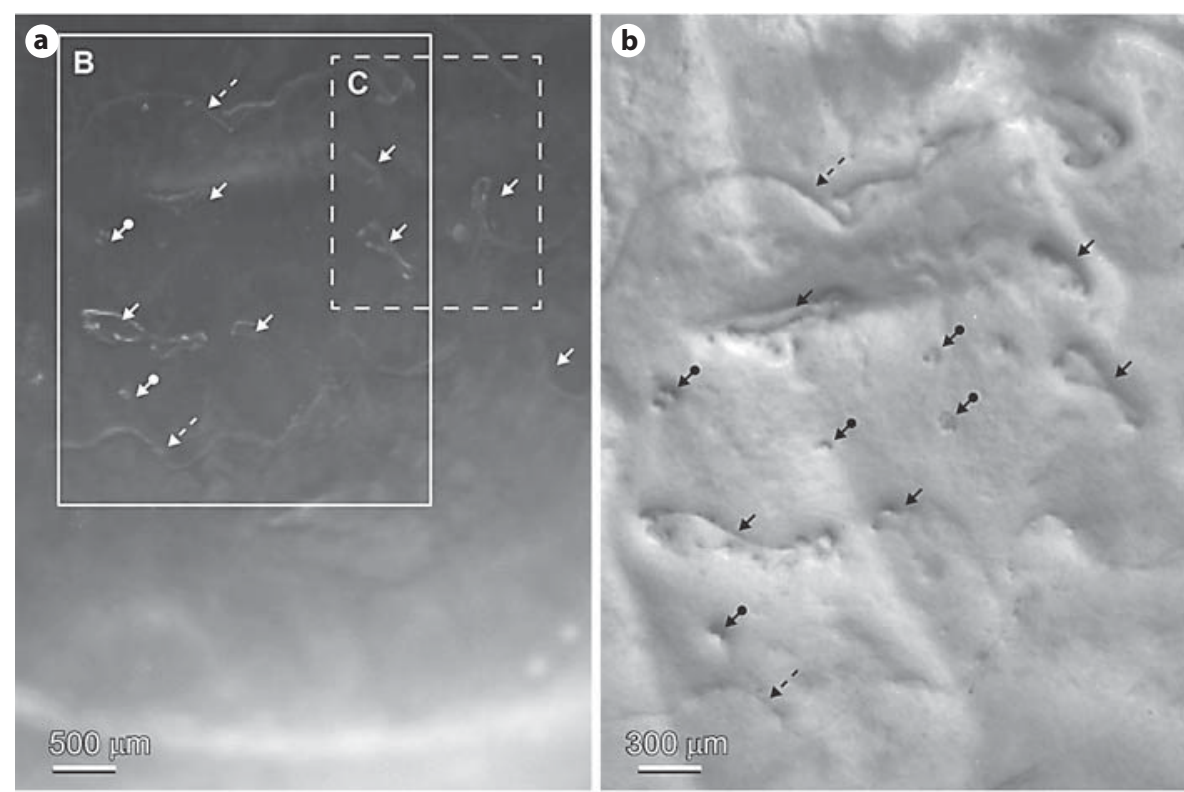

Fig. 1. Stereomicroscope images of the right cornea with diagnosed EBMD. a Dark-field illumination at a low magnification showed that numerous irregular geographic-shaped, faint gray-white patches were present in the central area of the inferior quadrants of the cornea. b Highly oblique illumination of the large framed area in a showed that the pathological lesions were present as fingerprints (arrows), dots (arrows with circles at the end) and ridge-like patterns (dashed arrows). Dark-field illumination (c) and oblique illumination (d) of the central area - dashed frame in $\mathbf{a}$ - in higher magnification showed the details of four fingerprint lesions.
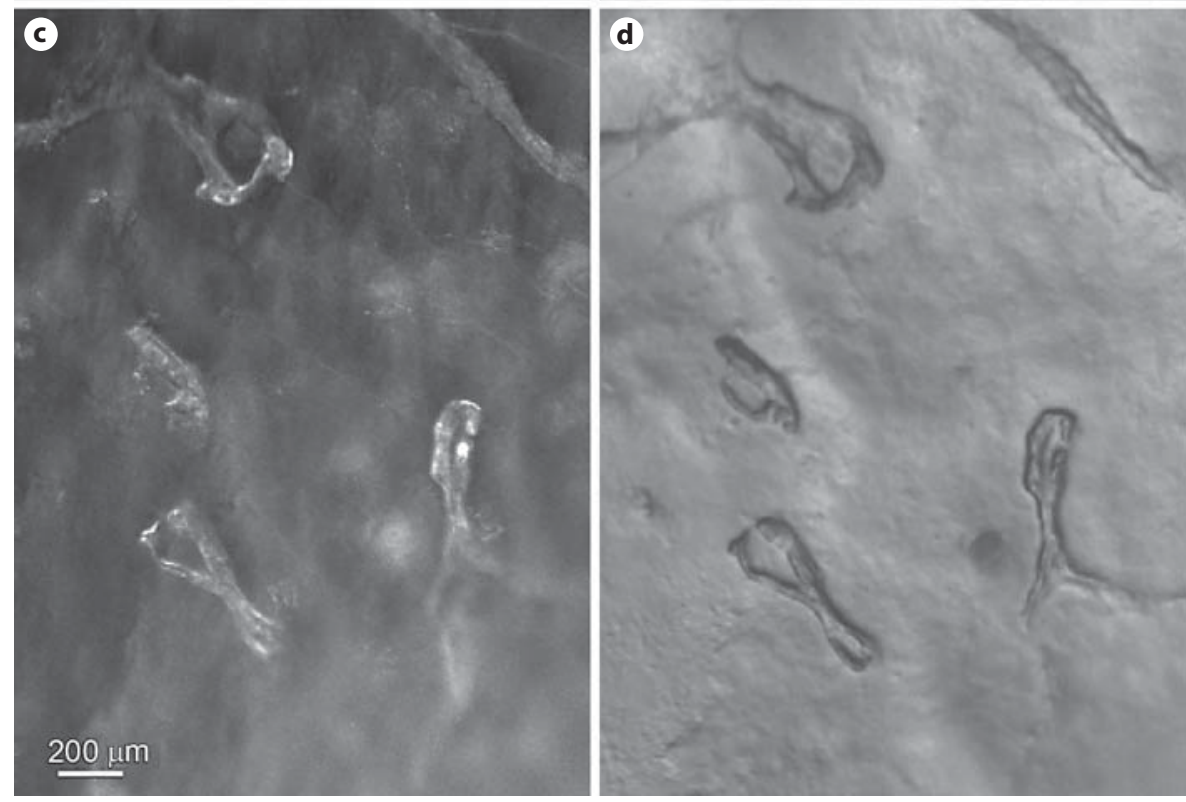

but it can be unilateral or very asymmetric in presentation [31]. In the current study, we used a recently developed and modified method of immunofluorescence and imaging to examine the nerve architecture in a unilateral EBMD cornea and to compare it with the normal eye from the same donor. The results demonstrate that EBMD remarkably changes the corneal epithelial nerve architecture, and significantly decreases epithelial nerve density and nerve terminals without altering the stromal nerves. In agreement with our results, a previous study using in vivo confocal microscopy has shown damaged subbasal nerves in EBMD patients, but this study limited examination to a small area of the cornea [23]. Although the exact mechanisms underlying this phenomenon remain unclear, we attribute this reduction to the abnormalities of the basement membrane. As reported previously, central epithelial innervation is supplied by branches of the stromal nerves $[20,29]$. These stromal nerves penetrate the basement membrane into the epithelium at the peripheral cornea, forming long bundles. The long bundles run in the intra- or subepithelium from the periphery to the center, dividing into numerous smaller branches which 

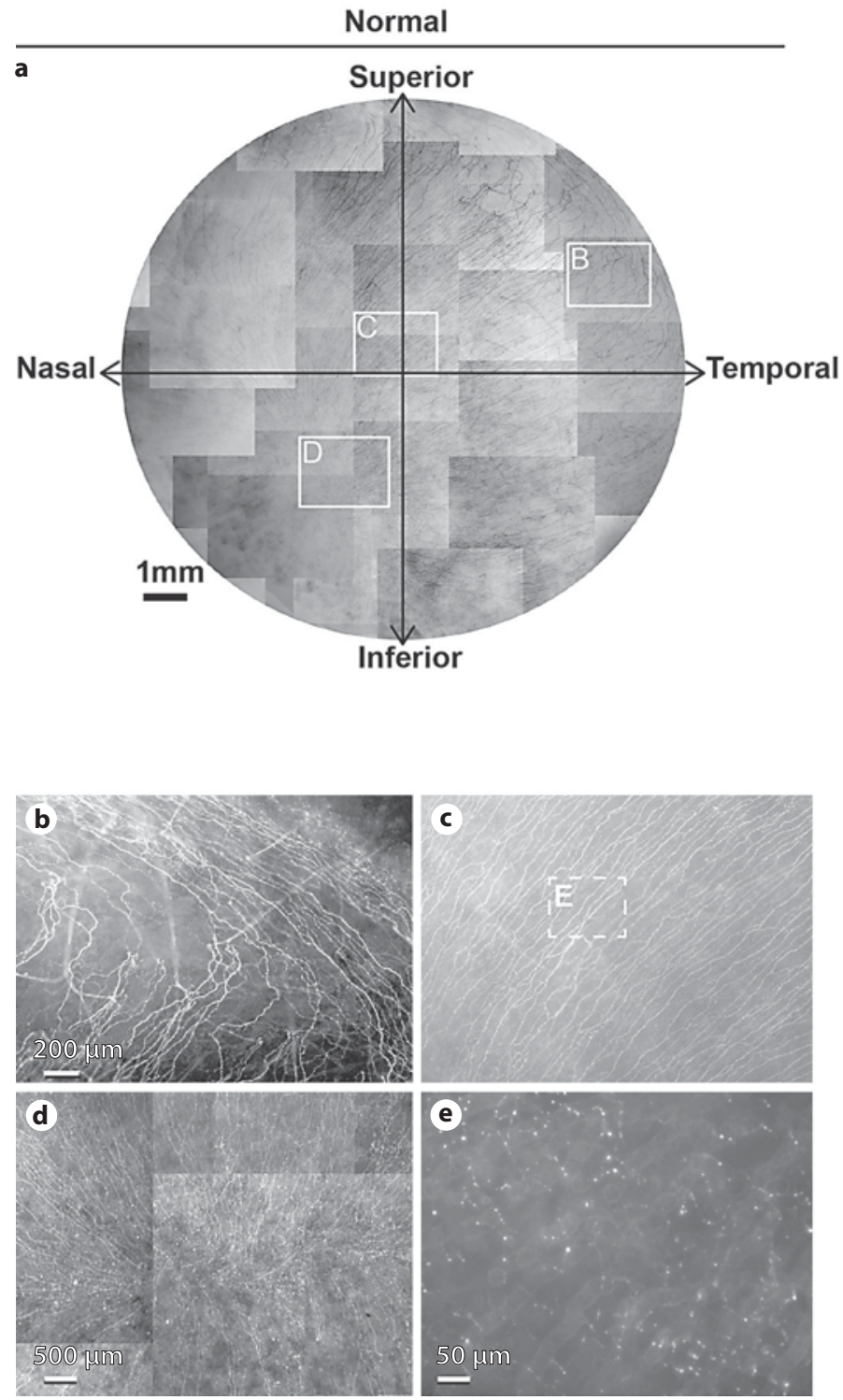

Fig. 2. a Whole mount view of epithelial nerve distribution in the normal left cornea. Images were acquired in a time lapse mode with a Nikon Eclipse TE200 and with a $5 \times$ lens in compliance with the natural shape of the cornea. The epithelial nerve bundles ran in a radial pattern from the periphery to merge in an area within the inferior nasal quadrant beyond the corneal apex. Highlighted images showed the detailed architecture of the epithelial nerve distribution in the peripheral (b), central (c) and vortex areas (d). e High magnification image recorded from the central area - as marked in c - shows the nerve terminals at the superficial epithelia. $\mathbf{f}$ Whole mount view of corneal epithelial nerve

\section{EBMD}
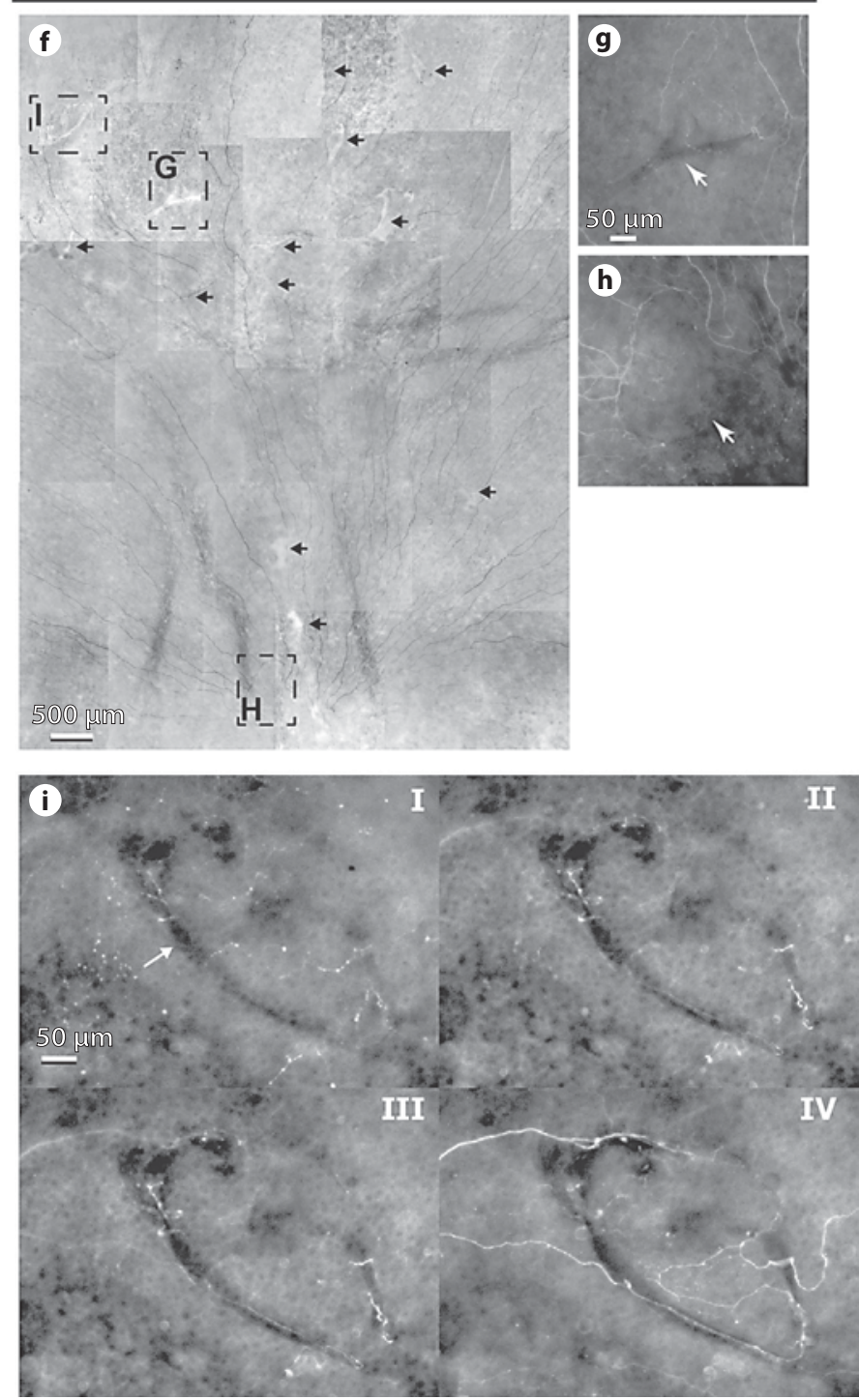

distribution in the central area of the inferior quadrant of the right cornea. The total area is about $45 \mathrm{~mm}^{2}$, consisting of $38 \mathrm{im}$ ages recorded in a time lapse mode with a $10 \times$ lens. Arrows indicate the pathological loci. g, $\mathbf{h}$ Highlighted images showing the detailed architecture of the epithelial nerve distribution in the central and vortex area as marked in f. i Topographic images recorded at the plane of superficial epithelia (I), wing cells (II), basal cells (III) and the basement membrane (IV) show detailed epithelial innervation in a fingerprint lesion (arrow) at the central cornea. 

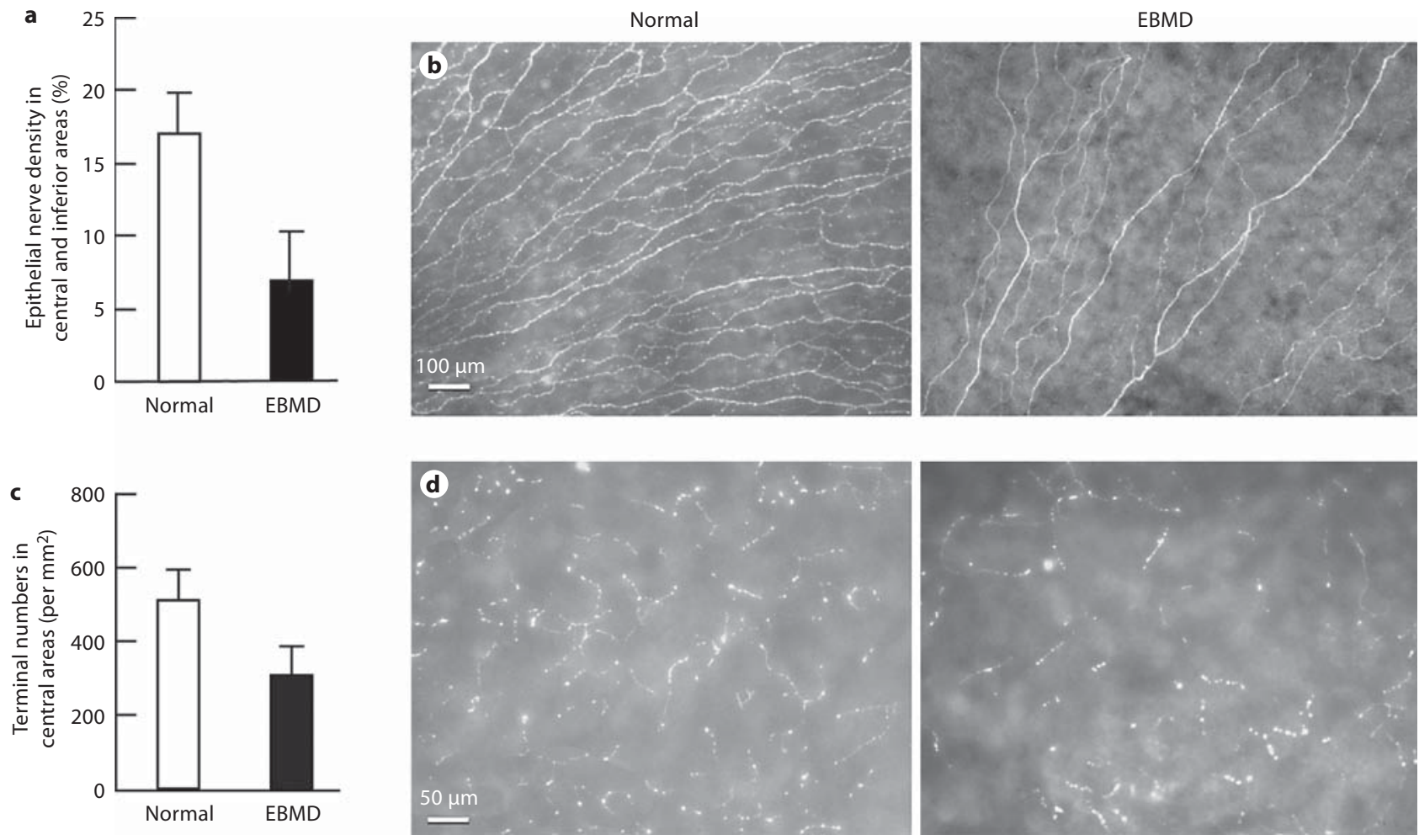

Fig. 3. Effect of EBMD on corneal subbasal nerve density and nerve terminals. a Subbasal nerve density in the central area of the inferior quadrant was calculated as percentage of total area in each image. A total of 36 images were taken at a magnification of $10 \times$ lens from each eye. These images were changed to grayscale mode and placed against a white background to get better contrast using Photoshop imaging software. The nerve fibers of each image were carefully drawn with 4-pixel lines following the course of each fiber. Percentage of nerve area was quantified for each im-

connect to each other and constitute a delicate nerve network within the epithelium. Fine nerve terminals or free endings budding from the network innervate the epithelial cells [29]. Early studies by light and electron microscopes have demonstrated that the basic histological finding in EBMD is a faulty basement membrane, which is thickened, multilaminar, and misdirected into the epithelium $[13,19,22]$. This aberrant basement membrane may prevent the passage of nerve fibers and change the course of nerve bundles, thus leading to a decrease of epithelial innervation in the pathological areas, as we observed in the present study.

EBMD is one of the most common corneal disorders and a major cause of recurrent erosions.

Corneal Nerves in Epithelial Basement Membrane Dystrophy age using the image analysis program. Data are expressed as average \pm SD. b Representative images of EBMD and normal subbasal nerves. c Number of nerve terminals. 20 images for each eye from the central corneas were used. Nerve terminals in superficial epithelia in each image were counted. Because each image took up an area of $0.335 \mathrm{~mm}^{2}$, the terminal numbers per $\mathrm{mm}^{2}$ were calculated. Data are expressed as average \pm SD. d Representative images of nerve terminals in both eyes.

Patients with EBMD are predisposed to epithelial sloughing/defects during the microkeratome pass in laser in situ keratomileusis, subsequent wound-healing problems, and multiple complications in the postoperative period [32].The basis for recurrent erosions is thought to be anomalies at the junction between basal epithelial cells and Bowman's layer, involving the epithelial basement membrane and hemidesmosomes of basal cells $[4,16]$, but the exact relationship between recurrent erosion and EBMD is not fully understood. In the current study, there are no available data about the donor's history of corneal symptoms, but our findings show that there is a significant decrease in epithelial nerve density and nerve terminals in the EBMD eye. 
Treatments for symptomatic EBMD include topical lubricants and hypertonic medications, contact lenses, or surgical procedure such as stromal puncture with a needle [33-35]. When the epithelial erosions are frequent, topical corticoids and inhibitors of metalloproteinase-9 have been effective in some cases [36]. In more severe cases, mechanical debridement of epithelium is recommended. Excimer laser phototherapeutic keratectomy is another technique to remove abnormal epithelial basement membrane [37-39]. These treatments cause pain and discomfort until epithelium regrowth, but a retrospective analysis of patients with recurrent erosions show that surgical treatments were associated with recurrences [7].

Corneal nerves serve a protective function and regulate corneal epithelial integrity, proliferation, and wound healing. To our knowledge, there is no study reporting changes in corneal sensitivity in EBDM patients. Therefore, we can only speculate that the significant reduction in epithelial nerves as shown in this study will decrease sensitivity and will induce dry eye, which might explain the recurrent erosions and the slower closure of epithelial defects after surgery in patients with EBMD. In that re- spect, our recent experimental studies in rabbits have shown restoration of nerve density and nerve functionality after lamellar keratectomy when the animals were treated with pigment epithelial-derived factor in conjunction with the omega-3 fatty acid, docosahexaenoic acid [40-42]. New treatment regimes that promote corneal nerve regeneration may be useful in improving the symptoms of EBMD patients.

\section{Acknowledgments}

This study was supported by R01EY019465 from the National Eye Institute. The content is solely the responsibility of the authors and does not necessarily represent the official view of the National Eye Institute or the National Institutes of Health. The study protocol of this research conforms to the Louisiana State University guidelines for ethical human study and has received support from the institution's board on human research, though no live participants were used and all tissue was donated.

\section{Disclosure Statement}

The authors declare that they have no conflicts of interest.

\section{References}

1 Vogt A: Lehrbuch und Atlas der Spaltlampenmikroskopie des lebenden Auges. Berlin, Springer, 1930, part 1, pp 264-265.

- 2 Cogan DG, Donaldson DD, Kuwabara T, Marshall D: Microcystic dystrophy of the corneal epithelium. Trans Am Ophthalmol Soc 1964;62:213-225.

3 Guerry D 3rd: Observations on Cogan's microcystic dystrophy of the corneal epithelium. Trans Am Ophthalmol Soc 1965;63: 320-334.

4 Rodrigues MM, Fine BS, Laibson PR, Zimmermann LE: Disorders of the corneal epithelium. A clinicopathologic study of dot, geographic, and fingerprint patterns. Arch Ophthalmol 1974;92:475-482.

$\checkmark 5$ Werblin TP, Hirst LW, Stark WJ, et al: Prevalence of map-dot-fingerprint changes in the cornea. Br J Ophthalmol 1981;65:401-409.

-6 Ghosh M, McCulloch C: Recurrent corneal erosion, microcystic epithelial dystrophy, map configurations and fingerprint lines in the cornea. Can J Ophthalmol 1986;21:246-252.

$\checkmark 7$ Reidy JJ, Paulus MP, Gona S: Recurrent erosions of the cornea: epidemiology and treatment. Cornea 2000;19:767-771.

8 Cogan DG, Kuwabara T, Donaldson DD, Collins E: Microcystic dystrophy of the cornea: a partial explanation for its pathogenesis. Arch Ophthalmol 1974;92:470-474.
$\$ 9$ Ehlers N, Møller HU: Pathology and pathomechanisms of epithelial microcystic and basement membrane abnormalities of the cornea. Acta Ophthalmol (Copenh) 1988;66:318-326.

10 Tripathi RC, Bron AJ: Cystic disorders of the corneal epithelium. II. Pathogenesis. Br J Ophthalmol 1973;57:376-390.

11 Fogle JA, Kenyon KR, Stark WJ, Green WR: Defective epithelial adhesion in anterior corneal dystrophies. Am J Ophthalmol 1975;79: 925-940.

12 Laibson PR: Microcystic corneal dystrophy. Trans Am Ophthalmol Soc 1976;74:488-531.

13 Brodrick JD, Dark AJ, Peace GW: Fingerprint dystrophy of the cornea: a histologic study. Arch Ophthalmol 1974;92:483-489.

14 Kaufman HE, Clower JW: Irregularities of Bowman's membrane. Am J Ophthalmol 1966;61:227-230.

15 Laibson PR, Krachmer JHP: Familial occurrence of dot (microcystic), map, fingerprint dystrophy of the cornea. Invest Ophthalmol Vis Sci 1975;14:397-399.

16 Fogle JA, Kenyon KR, Stark WJ, Green WR: Defective epithelial adhesion in anterior corneal dystrophies. Am J Ophthalmol 1975;79: 925-940.

17 Brown N, Bron A: Recurrent erosion of the cornea. Br J Ophthalmol 1976;60:84-96.
18 Kenyon KR: Recurrent corneal erosion pathogenesis and therapy. Int Ophthalmol Clin 1979;19:169-195.

19 Aitken DA, Zeidoon AB, Lee WR: Ultrastructural study of the corneal epithelium in the recurrent erosion syndrome. Br J Ophthalmol 1995;79:282-289.

20 Müller LJ, Marfurt CF, Kruse F, Tervo TM Corneal nerves: structure, content and function. Exp Eye Res 2003;76:521-542.

-21 Lambiase A, Bonini S, Micera A, et al: Expression of nerve growth factor receptors on the ocular surface in healthy subjects and during manifestation of inflammatory diseases. Invest Ophthalmol Vis Sci 1998;39: 1272-1275.

22 Akhtar S, Bron AJ, Meek KM, and Voon LW: Clinical and ultrastructural findings in mare's tail lines of the corneal epithelium. $\mathrm{Br}$ J Ophthalmol 2004;88:864-867.

23 Rosenberg ME, Tervo TM, Petroll WM, Vesaluoma $\mathrm{MH}$ : In vivo confocal microscopy of patients with corneal recurrent erosion syndrome or epithelial basement membrane dystrophy. Ophthalmology 2000;107:565-573.

24 Cavanagh HD, Petroll WM, Alizadeh H, et al: Clinical and diagnostic use of in vivo confocal microscopy in patients with corneal disease. Ophthalmology 1993;100:14441454. 
25 Kaufman SC, Beuerman RW, Kaufman HE: Diagnosis of advanced Fuchs' endothelial dystrophy with the confocal microscope. Am J Ophthalmol 1993;116:652:653.

-26 Mustonen RK, McDonald MB, Srivannaboon S, et al: In vivo confocal microscopy of Fuchs' endothelial dystrophy. Cornea 1998; 17:493-503.

27 Hernández-Quintela E, Mayer F, Dighiero P, et al: Confocal microscopy of cystic disorders of the corneal epithelium. Ophthalmology 1998;105:631-636.

28 Labbé A, Nicola RD, Dupas B, et al: Epithelial basement membrane dystrophy: evaluation with the HRT II Rostock Cornea Module. Ophthalmology 2006;113:1301-1308.

29 He J, Bazan NG, Bazan HE: Mapping the entire human corneal nerve architecture. Exp Eye Res 2010;91:513-523.

-30 He J, Bazan HE: Mapping the nerve architecture of diabetic human corneas. Ophthalmology 2012;119:956-964.

31 Weiss JS, Moller HU, Lisch W, et al: The IC3D classification of the corneal dystrophies. Cornea 2008;27(suppl 2):S1-S42.
32 Dastgheib KA, Clinch TE, Manche EE, et al: Sloughing of corneal epithelium and wound healing complications associated with laser in situ keratomileusis in patients with epithelial basement membrane dystrophy. Am J Ophthalmol 2000;130:297303.

33 Mclean EN, MacRae SM, Rich LF: Recurrent erosion: treatment by anterior stromal puncture. Ophthalmology 1986;93:784-788.

34 Buxton JN, Constad WH: Superficial epithelial keratectomy for map-dot-fingerprint corneal dystrophy. Ann Ophthalmol 1987; 19:92-96.

35 Hykin PG, Foss AE, Pavesio C, Dart JK: The natural history and management of recurrent corneal erosion: a prospective randomized trial. Eye 1994;8(pt 1):35-40.

36 Dursun D, Kim MC, Solomon A, et al: Treatment of recalcitrant recurrent corneal erosions with inhibitors of matrix metalloproteinase-doxycycline and corticosteroids. Am J Ophthalmol 2001;132:8-13.

37 Itty S, Hamilton SS, Baratz KH, et al: Outcomes of epithelial debridement for anterior basement membrane dystrophy. Am J Ophthalmol 2007; 144:288-289.
38 Sridhar MS, Rapuano CJ, Cosar CB, et al: PTK versus diamond burr polishing of Bowman's membrane in the treatment of recurrent corneal erosions associated with anterior basement membrane dystrophy. Ophthalmology 2002;109:674-679.

-39 Cavanaugh TB, Lind DM, Cutarelli PE, et al: Phototherapeutic keratectomy for recurrent erosion syndrome in anterior basement membrane dystrophy. Ophthalmology 1999; 106:971-976.

40 Cortina MS, He J, Li N, et al: Neuroprotectin D1 synthesis and corneal nerve regeneration after experimental surgery and treatment with PEDF plus DHA. Invest Ophthalmol Vis Sci 2010;51:804-810.

41 Cortina MS, He J, Li N, et al: Recovery of corneal sensitivity, calcitonin gene-related peptide-positive nerves and increased wound healing induced by pigment epithelial-derived factor plus docosahexaenoic acid after experimental surgery. Arch Ophthalmol 2012;130:76-83.

42 He J, Bazan HE: Omega-3 fatty acids in dry eye and corneal nerve regeneration after refractive surgery. Prostaglandins Leukot Essent Fatty Acids 2010;82:319-325. 